\section{DESENVOLVIMENTO DE TESTES PARA AVALIAÇÃO DE VELOCIDADE E AGILIDADE DE JOGADORES DE VOLEIBOL SENTADO}

Título resumido:

Velocidade e agilidade de jogadores de voleibol sentado

\author{
Saulo Oliveira ${ }^{1}$; Inês Oliveira ${ }^{2}$; Ronaldo Severien ${ }^{2}$; Ery Araújo ${ }^{2}$; Josiete Santos ${ }^{1}$; \\ Ramon Milano ${ }^{1}$; Manoel Costa ${ }^{3}$
}

\title{
RESUMO
}

Objetivo: Nosso objetivo foi desenvolver e validar testes para avaliação da velocidade e agilidade de jogadores de voleibol sentado. Método: A criação foi baseada nas características da modalidade (deslocamento sentado com três apoios e utilização dos braços e do quadril). A investigação teve três fases de análise: a) avaliação por experts; b) objetividade e reprodutibilidade teste-reteste (sete dias); e c) validade discriminante. Fase 1: cinco experts assistiram a vídeos de jogadores executando os testes e responderam um formulário baseado em escala Likert de cinco pontos. Fase 2: seis jogadores experientes executaram os testes e foram avaliados por três pesquisadores independentes, em um intervalo de sete dias. Fase 3: os atletas foram comparados a seis congêneres destreinados. Para objetividade foi usado o teste de Wilcoxon. Quanto à reprodutibilidade, optamos pelo coeficiente de correlação intraclasse. Para a validade discriminante, consideramos o teste U-Mann Whitney. Observaram-se níveis excelentes de validade de conteúdo (ICV: 0,96). Resultados: Os três avaliadores apresentaram objetividade satisfatória para a velocidade (avaliador A: 4,20s; avaliador B: 3,96s; avaliador C: $3,98 \mathrm{~s} ; \mathrm{p}=0,301$ ) e para agilidade (avaliador A: 14,41s; avaliador B: 13,24; avaliador C: 13,52; $p=0,134$ ). A reprodutibilidade foi considerada excelente para a velocidade $(\mathrm{CCl}: 0,96 ; p<0.001)$ e agilidade $(\mathrm{CCl}$ : 0,79 ; $\mathrm{p}=0,052$ ). Ao compararmos com um grupo destreinado, os jogadores apresentaram melhores resultados para a velocidade $(3,72 \mathrm{~s}$ contra $5,89 \mathrm{~s} ; \mathrm{p}=0,002)$ e agilidade $(12,78 \mathrm{~s}$ contra $15,74 \mathrm{~s} ; \mathrm{p}=0,002)$. Conclusão: Concluímos que os testes são válidos e sensíveis para controle, acompanhamento e avaliação de atletas da modalidade, sendo uma ferramenta viável para aplicação prática do treinamento esportivo.

Palavras-chave: Pessoas com deficiência, esporte, treinamento esportivo.

\section{ABSTRACT}

Objective: Our goal was to develop and validate tests to assess the speed and agility of sitting volleyball players. Method: The creation was based on the characteristics of the modality (seated displacement with three supports and use of arms and hip). The research had three phases of analysis: a) evaluation by experts; B) test-retest objectivity and reproducibility (seven days); And c) discriminant validity. Phase 1: Five experts watched videos of players running the tests and answered a form based on a five-point Likert scale. Phase 2: Six experienced players performed the tests and were evaluated by three independent researchers within a seven-day interval. Phase 3: athletes were compared to six untrained congeners. The Kruskal-Walis test was used for objectivity. Regarding reproducibility, we chose the intraclass correlation coefficient. For the discriminant validity, we consider the U-Mann Whitney test. Excellent levels of content validity (ICV: 0.96) were observed. Results: The three evaluators presented satisfactory objectivity for the velocity (evaluator $A: 4,20$ s, evaluator $B: 3,96$ s, evaluator $C$ : $3,98 \mathrm{~s}, \mathrm{p}=0,301$ ) and for agility (evaluator $\mathrm{A}: 14,41 \mathrm{~s}$, evaluator $\mathrm{B}: 13,24$, evaluator $\mathrm{C}: 13.52, \mathrm{p}=0.134$ ). Reproducibility was considered excellent for speed (ICC: $0.96, p<0.001$ ) and agility (ICC: $0.79, p=0.052$ ). When compared to an untrained group, the players presented better results for speed (3.72s versus $5.89 \mathrm{~s}, p=0.002$ ) and agility (12.78s versus $15.74 \mathrm{~s}, \mathrm{p}=0.002)$. Conclusion: We conclude that the tests are valid and sensitive for the control, monitoring and evaluation of athletes of the sport, being a possible tool to be applied in the practice of sports training.

Keywords: People with disabilities, sport, Sports training.

1 Universidade Federal de Pernambuco, Centro Acadêmico de Vitoria, Núcleo de Educação Física e Ciências do Esporte, Vitoria de Santo Antão, Pernambuco, Brasil.

e-mail: saulofmoliveira@gmail.com; josiete-maria@bol.com.br; ramon_atroch@hotmail.com

2 Universidade de Pernambuco, Escola Superior de Educação Física, Recife, Pernambuco, Brasil. e-mail: inesgloliveira@gmail.com; ronaldo.severien@hotmail.com; ery-araujo@hotmail.com

3 Universidade de Pernambuco, Laboratório de Avaliação da Performance Humana, Recife, Pernambuco, Brasil. e-mail: mcosta2@gmail.com

Endereço para correspondência: Iberê Caldas Souza Leão, Universidade Federal de Pernambuco - Centro Acadêmico de Vitoria, Núcleo de Educação Física e Ciências do Esporte. Rua: Alto do Reservatório, S/N, Bela Vista CEP: 55608-680 - Vitoria de Santo Antão, PE - Brasil. Fone: 81-35233351 / 997746783. E-mail: iberecaldas@gmail.com 


\section{INTRODUÇÃO}

Deficiência vem a ser uma perda, carência ou anormalidade de estrutura ou função psicológica, fisiológica ou anatômica, temporária ou permanente. Incluem-se nessas a incidência de uma anomalia, defeito ou perda de um membro, órgão, tecido ou qualquer outra estrutura do corpo, inclusive das funções mentais e neurais (CHAN e ZOELLICK, 2011).

No Brasil, o desenvolvimento do esporte para pessoas portadoras de deficiência física teve início a partir de 1958 com a elaboração da fundação do Clube dos Paraplégicos em São Paulo e do Clube do Otimismo no Rio de Janeiro. No fim da década de 50 , os educadores físicos começaram a ter uma visão diferenciada e mais focada para esse público com o início das atividades adaptadas. Sendo introduzidas no meio desportivo através de programas médicos que tinham como fins a prevenção de doenças e reabilitação para a população portadora de deficiência física ou motora (PEDRINELLI, 1994; ADAMS et al., 1985).

O esporte adaptado pode ser contextualizado como terapêutico, recreacional e de alto rendimento, tendo uma contribuição com 0 desenvolvimento da autoestima, da autoconfiança, das condições e capacidades físicas de seus praticantes, atuando como um estímulo positivo em relação à auto-imagem, independência, superação e ainda prevenindo deficiências secundárias ((BRAZUNA e CASTRO, 2011).

Nesse contexto, o voleibol sentado surgiu em 1956 na Holanda e teve sua estreia nos Jogos Paralímpicos em 1976 (Toronto, Canadá) como esporte de exibição. Em 1980 é incorporado ao programa oficial dos Jogos Paralímpicos e iniciou suas atividades no Brasil em 2002 (MELO e WINCKLER, 2012). Um esporte adaptado que surgiu partir da união do voleibol convencional com o Sitzball, o qual era jogado sentado no chão e sem rede. Até as Paraolimpíadas de Sydney (2000) o voleibol sentado era dividido em duas categorias, sendo elas, sentado e em pé. A partir dos jogos de Atenas 2004, passou a ser disputado apenas sentado, e tornou-se permitida a participação das mulheres (CPB, 2017).

A tecnologia vem evoluindo e acompanhando o voleibol convencional, mas no voleibol sentado, por ser uma modalidade criada bem recente, todas as informações produzidas são ainda baseadas pelo conhecimento informal dos integrantes da comissão técnica e atletas o que vem dificultando a produção de informações precisas, relevantes e confiáveis (COLLET et al., 2011). Quando se é produzido indicadores de rendimento que venham a trazer delineamento de modelos competitivos de jogos, tanto os profissionais como os atletas poderão ter um entendimento mais preciso e dinâmico da modalidade, dando assim um norte para a elaboração de planejamentos, rotinas de treinamento físico e tático. Tendo em vista o déficit de parâmetros de avaliação para atletas de voleibol sentado o presente estudo tem como objetivo desenvolver e validar testes específicos para jogadores da modalidade, que visam medir a agilidade e velocidade através de um teste fidedigno e de fácil aplicabilidade buscando proximidade com a modalidade.

\section{MATERIAIS E MÉTODOS \\ Pesquisa e delineamento}

Esta pesquisa trata-se de uma pesquisa de caráter descritivo comparativo, protocolada aprovada pelo Comitê de Ética em Pesquisa com Seres Humanos da Universidade de Pernambuco, sob o número 078/11. Após a criação dos protocolos, com base na dimensão da quadra e do tipo de deslocamento dos atletas, a pesquisa foi dividida em três etapas: a) validação por experts; b) verificação da reprodutibilidade e objetividade; e c) validação discriminante. Inicialmente os testes foram desenvolvidos utilizando como parâmetros a maneira de deslocamento na quadra de jogo dos atletas, bem como as dimensões da quadra. Adiante uma descrição dos protocolos é apresentada, com ilustrações para visualizar a sua maneira de execução.

Teste de velocidade em 8 metros (V8m): posicionar o atleta com o quadril atrás da linha de saída. Ao sinal do avaliador ele deverá percorrer na sua maior velocidade a distancia estabelecida em direção ao terceiro cone. $O$ avaliador acionará o cronômetro no primeiro movimento do atleta e desligará após o mesmo ultrapassar com o quadril a linha do segundo cone cujo objetivo será percorrer a distância estabelecida no menor tempo entre duas tentativas.

Teste de agilidade em cruz (TACRUZ): posicionar o atleta no centro do percurso demarcado em forma de cruz por cones com distâncias iguais a 2,5 metros. Ao sinal do avaliador ele deve se deslocar em direção ao primeiro cone à sua frente, contorná-lo e em seguida retornar de costas ao ponto central. Após o seu retorno, o mesmo percurso deverá ser realizado em direção ao cone posicionado às suas costas, que também deverá ser contornado com retorno de frente ao ponto central. Em seguida, o avaliado deverá deslocar-se lateralmente em direção ao cone localizado no seu lado direito, contornando-o e retornando novamente ao ponto central. O ultimo percurso deverá ser realizado também lateralmente, em direção ao cone à sua esquerda, para contorná-lo com retorno em seguida ao ponto central. O cronômetro é acionado pelo avaliador ao primeiro movimento do avaliado á sua frente e desligado após o retorno do ultimo cone. $\mathrm{Na}$ figura 1 são apresentadas ilustrações dos protocolos desenvolvidos. 


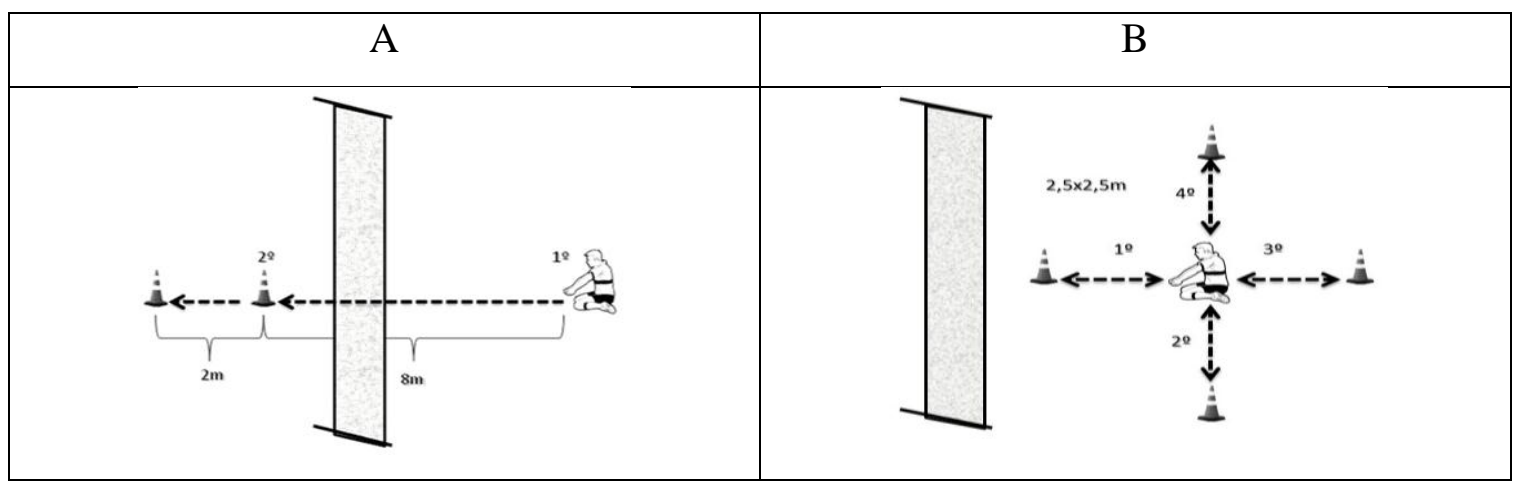

Figura 1. Ilustrações dos testes de velocidade (A) e agilidade (B) para avaliação de jogadores de voleibol sentado

\section{Estudo 1: validação por experts}

No primeiro momento foi realizado o processo de validação dos testes de cruz e $8 \mathrm{~m}$, para esse processo de validação arquivos foram criados (questionário contendo cinco questões baseadas na escala de Lickert). Que juntamente com os vídeos contendo cenas dos atletas de voleibol sentado executando os testes de agilidade e velocidade que foram mostrados para banca examinadora. Foram convidados cinco professores de educação física do estado de Pernambuco especialistas em avaliação e com proximidade com esportes coletivos através contato pessoal para participarem do teste de maneira voluntária, foi assinado o termo de consentimento livre e esclarecido. Contamos com a experiência de profissionais da área para elaboração dos testes, levando em consideração a importância das capacidades envolvidas de acordo com as exigências da modalidade.

O critério de inclusão e exclusão adotados para esse primeiro momento foram, para ser incluso teriam que ser professores/treinadores com o mínimo de dois anos de experiência no âmbito da prática de atividade física/esportes, por parte de pessoas com algum tipo de deficiência, além de ter experiência na área de avaliação do desempenho atlético. Para a exclusão, foram excluídos aqueles profissionais que não responderam ao questionário de maneira correta, não compreenderam $o$ processo de análise e respostas do questionário de validação e atrasaram em mais de quinze dias o envio das respostas.

\section{Estudo 2: reprodutibilidade e objetividade}

Foi realizada a verificação dos pressupostos de autenticidade científica de reprodutibilidade e objetividade dos testes de $8 \mathrm{~m}$ e cruz, para a verificação foram aplicados os devidos testes da seguinte maneira. $O$ ambiente de aplicação foi o mesmo no qual os atletas realizam seus treinos (quadra com solo em cimento), os avaliados foram 6 praticantes de voleibol sentado, com média de prática da modalidade de aproximadamente 07 anos, a coleta foi dividida em duas partes, sendo a primeira o teste e a segunda o reteste, o reteste foi aplicado entre um tempo superior a 2 dias e inferior a 7 dias. Antes de serem submetidos a realização do teste, os atletas passaram por um reconhecimento do mesmo no devido local de aplicabilidade, foram utilizados três avaliadores devidamente treinados no qual se revezaram na administração do teste, o teste inicial serviu para verificar a objetividade, já o reteste vem verificar a reprodutibilidade.
Os testes foram elaborados por dois experts da área que já vinham a mais de dois anos na prática de treinamento esportivo com o público deficiente e na modalidade de voleibol. Além desse critério foram utilizados os critérios que vinham a trazer o teste o mais próximo da realidade, como dimensões de quadra e movimentações dos atletas de acordo com a demanda prevista na modalidade buscando sempre a semelhança tanto da movimentação quando do esforço exercido pelos atletas.

\section{Estudo 3: validação discriminante}

Foi realizada a verificação da validade discriminante dos testes de $8 \mathrm{~m}$ e cruz, onde participaram do estudo 12 sujeitos, sendo divididos em seis jogadores de voleibol sentado, com experiência média de cinco anos de prática, e seis homens destreinados, mas fisicamente ativos.

$\mathrm{Na}$ primeira visita, os voluntários passaram por uma familiarização com a execução dos testes. Já segunda visita, após um intervalo de tempo de sete dias, os sujeitos foram avaliados novamente para que as medidas reais de agilidade e velocidade fossem consideradas. Para cada teste foram realizadas duas tentativas, sendo considerada sempre a que obtinha melhor medida de agilidade e velocidade. Todos os procedimentos foram realizados pela mesma equipe treinada.

\section{Análise Estatística}

Para as análises de dados da primeira fase do presente estudo foram utilizados o índice de validação de conteúdo. O ICV intende-se como um método muito utilizado na área de saúde para medir a proporção ou porcentagem de juízes que estão em concordância sobre determinados aspectos do instrumento e de seus itens. Permite inicialmente analisar cada item individualmente e depois o instrumento como um todo. Este modelo de avaliação vem a utilizar uma escala tipo Likert com pontuação de um a cinco. Para coleta das informações dos experts foi utilizada uma ficha apropriada para este fim, contendo itens que procuram identificar as percepções dos avaliadores sobre os testes desenvolvidos.

O escore do índice é calculado por meio da soma de concordância dos itens que foram marcados por "3" ou "4" pelos especialistas. Os itens que receberam pontuação "1" ou "2" devem ser revisados ou eliminados. Dessa forma, o IVC tem sido também definido como "a proporção de itens que recebe uma pontuação de 4 ou 5 pelos juízes". A fórmula para avaliar cada item individualmente fica assim: 
$I C V=\frac{N o \text { de respostas "4" ou "5" }}{N^{\circ} \text { total de respostas }}$

Para determinação da validade, foi considerado aceitável um valor de ICV maior que 0.75 , conforme indicações de Alexandre (8).

$\mathrm{Na}$ segunda fase do mesmo estudo foram adotados métodos de análises de dados diferente tendo em vista que tinham que avaliar a objetividade e reprodutibilidade do teste. Foram utilizados inicialmente para analisar a objetividade dos dois testes, o teste de comparações de postos de Wilcoxon. Para verificar a reprodutibilidade dos protocolos optou-se pela utilização do coeficiente de correlação de Spearman, da análise gráfica de BlandAltman e da comparação de postos de Wilcoxon. A escolha dos protocolos deu-se devido ao reduzido número de sujeitos na pesquisa, optou-se por realizar as análises considerando testes não-paramétricos.
Em todas as análises considerou-se significante um valor de $\mathrm{P}<0.05$

$\mathrm{Na}$ terceira e última fase do estudo foram utilizados verificação de pressupostos de normalidade, por meio do teste de Shapiro-Wilk. Em virtude do resultado de normalidade do teste, devido ao baixo número de sujeitos em ambos os grupos, optou-se por utilizar o teste $U$ de Mann-Withney para comparação dos resultados de agilidade e velocidade entre os grupos. Para todas as análises foi utilizado o software Prism, versão 6.0, e considerou-se um nível de significância de $5 \%(p<0.05)$.

\section{RESULTADOS}

Primeira fase: Na validação por expert inicialmente foram recolhidos alguns dados demográficos dos avaliadores, que estão presente na tabela abaixo.

Tabela 1. Dados demográficos dos avaliadores que participaram da pesquisa

\begin{tabular}{lllll}
\hline Avaliadores & $\begin{array}{l}\text { Idade } \\
\text { (Anos) }\end{array}$ & $\begin{array}{l}\text { Graduação } \\
\text { (Curso) }\end{array}$ & Tempo de experiência (Anos) & Sexo \\
\hline 1 & 34 & Ed.Física & 7 & $\mathrm{M}$ \\
2 & 51 & Ed.Física & 16 & $\mathrm{M}$ \\
3 & 26 & Ed.Física & 4 & $\mathrm{M}$ \\
4 & 25 & Ed.Física & 2 & $\mathrm{M}$ \\
5 & 41 & Ed.Física & 21 & $\mathrm{~F}$ \\
\hline
\end{tabular}

Ed.Física: educação física; M: masculino; F: feminino

Mais adiante após serem visualizados os vídeos e ser atribuídos às notas de cada expert foi elaborada a tabela com as notas mediante cada questão abordada.

Tabela 2. Respostas às questões formuladas após a visualização dos vídeos

\begin{tabular}{|c|c|c|}
\hline \multicolumn{2}{|c|}{ PERGUNTAS FORMULADAS } & RESPOSTAS* \\
\hline a) & $\begin{array}{l}\text { Sobre a cena analisada, a imagem é apresentada de maneira visualmente } \\
\text { clara? }\end{array}$ & 4,60 \\
\hline b) & $\begin{array}{l}\text { Você acha que no vídeo } 1 \text { (teste da cruz), a agilidade está sendo testada em } \\
\text { que medida? }\end{array}$ & 4,20 \\
\hline c) & $\begin{array}{l}\text { Você acha que no vídeo } 2 \text { (teste de sprint), a velocidade está sendo testada } \\
\text { em que medida? }\end{array}$ & 4,40 \\
\hline d) & $\begin{array}{l}\text { Sobre os dois testes, você acha que eles proporcionam AO ATLETA um } \\
\text { entendimento de sua execução em que medida? }\end{array}$ & 4,60 \\
\hline e) & $\begin{array}{l}\text { Sobre os dois testes analisados, você acha que eles proporcionam AO } \\
\text { TREINADOR facilidade em sua administração em que medida? }\end{array}$ & 5,00 \\
\hline
\end{tabular}

Legenda: *representa a média dos itens que foram respondidos pelos avaliadores. A escala Lickert variou de 1 ("nada", nas perguntas de [a] a [c]; e "difícil", nas perguntas [d] e [e]) a 5 ("totalmente", nas perguntas de [a] a [c]; e "fácil", nas perguntas [d] e [e]).

Como podemos analisar a tabela a pontuação atribuída foi entre 4 e 5 , portanto foi utilizado a metodologia proposta por Alexandre, sendo assim a verificação do índice de validade de conteúdo foi considerado aceitável com uma media de $(I C V=0,96)$, tendo em vista que $(I V C=0,75)$ foi a proposta valida mencionada por Alexandre e Coluci (ALEXANDRE e COLUCI, 2011).

Segunda fase: Na segunda fase foram analisadas a objetividade e reprodutibilidade, ao analisar os dados chegamos no seguinte resultado.

Para o teste de velocidade (Painel A) não foi observada diferença significante entre os três avaliadores. Contudo, para o teste de agilidade (Painel B), foi observada diferença significante apenas nas medidas do avaliador A e B. Percebeu-se que para os valores de velocidade (figura 2, A), a média da execução do teste diminuiu ao longo das três tentativas. Já para o teste de agilidade (figura $2, \mathrm{~B}$ ) houve uma queda entre a medida A e a medida B, e uma estabilização entre a medida B e a medida C. 


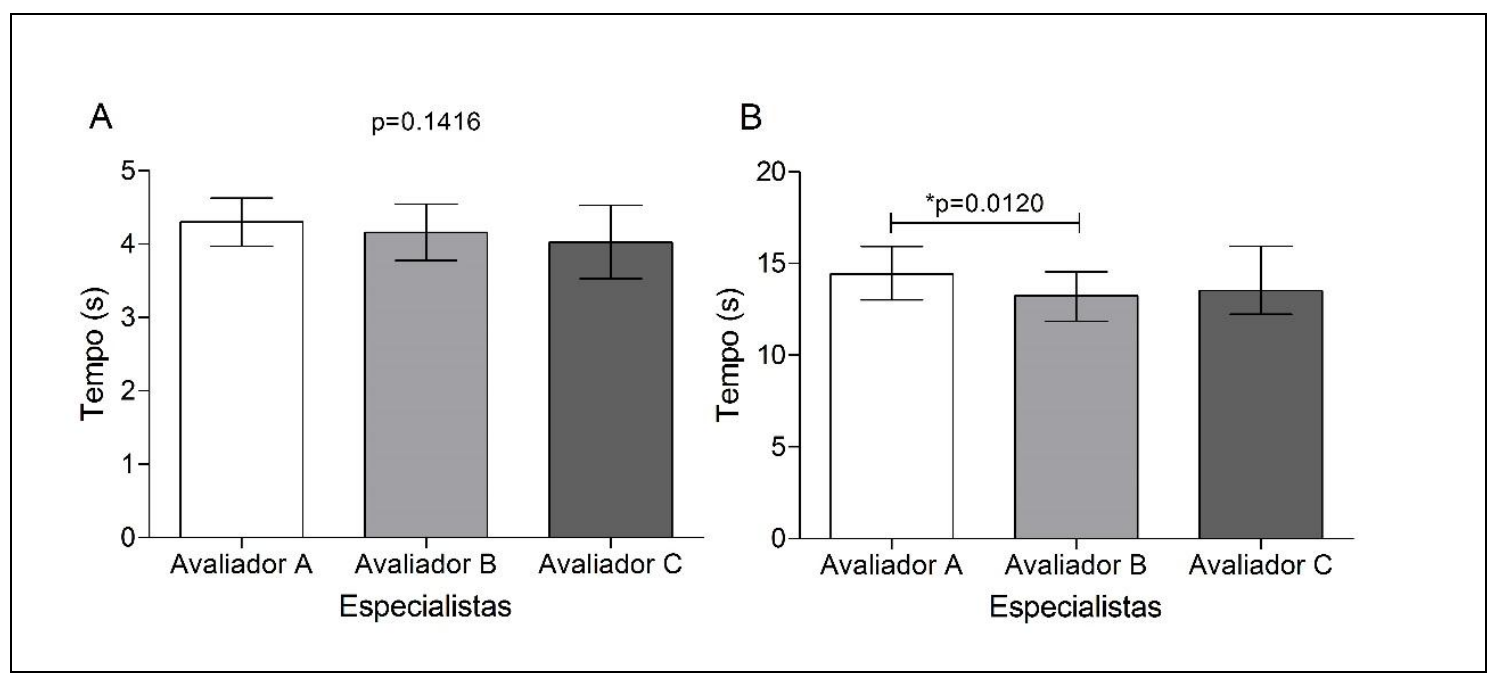

Figura 2. Análise da objetividade dos testes de velocidade (painel A) e agilidade (painel B) em jogadores de voleibol sentado; *Diferença estatisticamente significante.

No que concerne à avaliação da reprodutibilidade, os dados são apresentados na figura 3. Para ambos os testes não foram observadas diferenças significantes entre os dias de execução (figura $3, \mathrm{~A}$ e B).

A

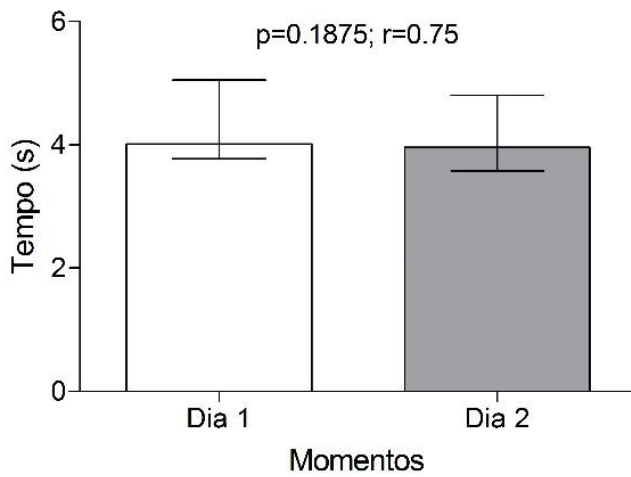

B

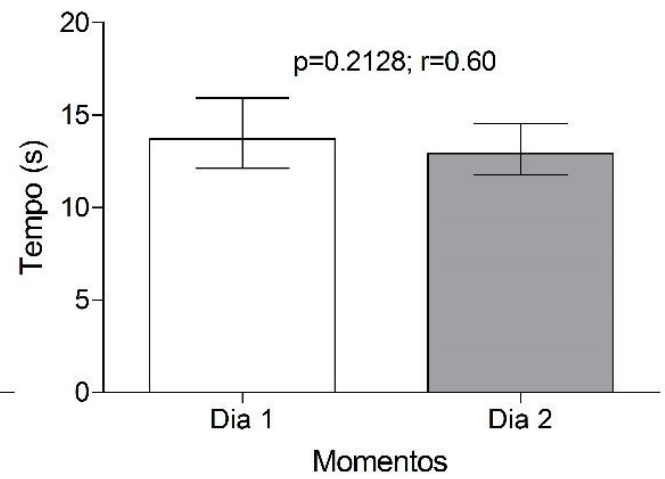

Figura 3. Análise da reprodutibilidade das medidas para os testes de velocidade (A) e agilidade (B); $p$ teste de Wilcoxon para medidas independentes; $r$ correlação de Spearman.

Analisando a relação entre as medidas, foi observado que o teste de velocidade apresenta uma correlação alta e valores concordantes pela análise gráfica de Bland-Altman na a execução teste-reteste (figura 4, painel A). Para o teste de agilidade, foi verificado que os valores de correlação entre as medidas 1 e 2 foi moderado, sendo as medidas concordantes (figura 4, painel B).

\section{A}

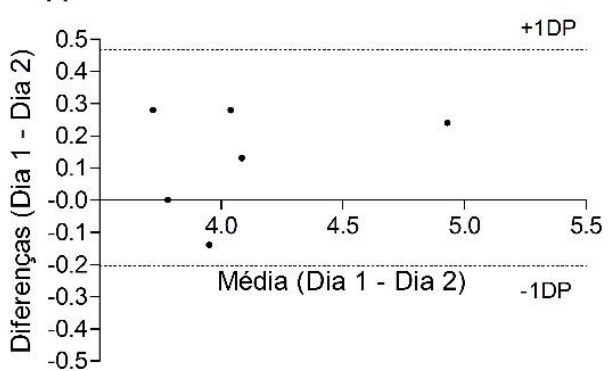

B

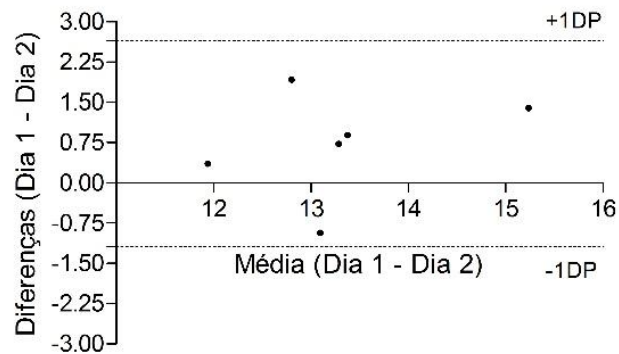

Figura 4. Análise gráfica de Bland-Altman entre as medidas do teste e do reteste para o protocolo de avaliação da velocidade (A) e da agilidade (B); +1DP limite superior de concordância determinado em 95\% de IC; -1DP limite de concordância inferior determinado em $95 \%$ de IC; todos os valores para ambos os testes se encontram dentro dos limites de concordância estabelecidos pelo método. 
Terceira fase: Na terceira fase foi analisado verificação da validade discriminante, levando em consideração aos valores medianos, observou-se que houve diferença estatisticamente significante entre os grupos para ambos os testes. O grupo de atletas apresentou valores de agilidade e velocidade sempre superiores aos obtidos pelo grupo de não atletas $(\mathrm{p}<0.05)$.

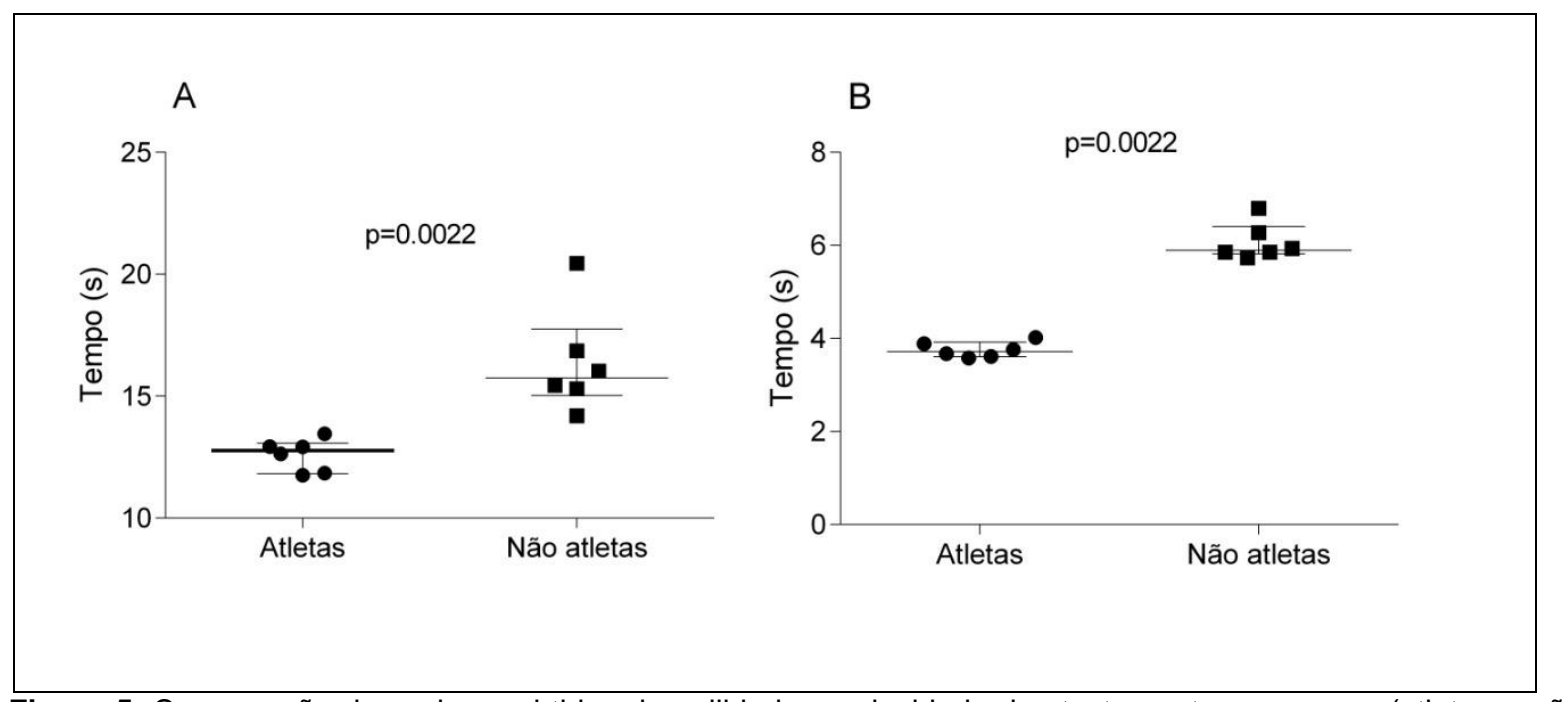

Figura 5. Comparação dos valores obtidos de agilidade e velocidade dos testes entre os grupos (atletas e não atletas). Foram observados valores superiores em ambos os testes para os atletas de voleibol sentado $(p<0.05)$

\section{DISCUSSÃO}

Objetivo do nosso estudo foi validar os testes de cruz e 8M, que visam medir a agilidade e velocidade através de um teste fidedigno e de fácil aplicabilidade buscando proximidade com a modalidade, tendo em vista o déficit de parâmetros de avaliação para atletas de voleibol sentado.

A elaboração desta pesquisa juntamente com outras correlacionadas à mesma área de atuação vem a somar buscando contribuir para 0 desenvolvimento do voleibol sentado em nosso país. O estudo trabalha de uma maneira particular as capacidades físicas exigidas por esse esporte, quanto esporte coletivo e suas demandas, sendo abordadas as capacidades agilidade e velocidade, que tem o propósito de servir como orientação para professores e treinadores na aplicação de testes, com a finalidade de mensurar e melhorar estas capacidades físicas em seus atletas.

Mediante os resultados positivos das três etapas do estudo, que vieram a ser bastante satisfatórias, mostrando assim 0 grau de reprodutibilidade e objetividade dos testes executados, tornando-os viáveis para profissionais de modalidades adaptadas.

No voleibol sentado, são poucas as ferramentas desenvolvidas para análise de uma habilidade específica para o jogo (MOLIK et al.,2008). Na investigação de Molik et al., testes de velocidade, potência e agilidade foram aplicados sugerindo uma bateria simples para a modalidade. Entretanto, a distância sugerida para o teste de agilidade é elevada, incompatível com as peculiaridades do voleibol sentado, uma vez que os autores utilizaram uma metragem adaptada do basquetebol. Além disso, estes autores não analisaram características psicométricas dos testes, limitando a discussão com o presente estudo.

Percebe-se, contudo, que outros fatores estão associados à agilidade, sobretudo, referente ao voleibol sentado. O posicionamento das mãos, por exemplo, é essencial não somente para melhorar a força de propulsão contra o solo e, por consequência, a velocidade do movimento, mas para fazer com que os atletas tenham reações mais rápidas e adequadas para coordenar as ações na realização do contato na bola, de acordo com a situação de jogo (SINGHAL et al., 2013).

Por tratar-se de uma modalidade que exige grande velocidade de deslocamento, associada as mudanças rápidas de direção, o voleibol sentado para o deficiente físico requer bom nível de força rápida (potência) de membros superiores, especialmente da musculatura específica envolvida na propulsão do deslocamento em três apoios vencendo 0 atrito com 0 solo. Além disso, a agilidade exerce elevada importância na modalidade esportiva, já que possibilita mudanças de direção sem perda de velocidade ou ritmo (WINNICK, 2004).

Em um estudo realizado com atletas de basquetebol em cadeira de rodas, Brasile propôs um teste de 20 metros de corrida máxima, para medir a capacidade máxima de deslocamento em velocidade dos atletas, embora este mecanismo não inclua a direção na trajetória, o mesmo é um dos poucos encontrados na literatura científica tratando sobre a avaliação de desempenho motor em atletas deste desporto (BRASILE, 1990).

Santos e Chagas adaptaram alguns testes neuromotores a fim de avaliar a evolução de atletas lesados medulares e amputados de membro inferior após dois meses de treinamento de basquetebol em cadeira de rodas. Porém nesta ocasião não foi testado a validade científica do mesmo, sendo assim os autores consideraram que fatores como tipo de quadra e cadeira de rodas podem ter interferido nos resultados (SANTOS e CHAGAS, 2001).

Tendo em vista a fácil aplicabilidade e reprodutibilidade dos testes, o mesmo vem a ser uma ferramenta muito importante em meio aos profissionais e treinadores envolvidos com modalidades adaptadas, por poder estar mensurando rendimento e qualidade dos atletas mediantes os treinos, podendo avaliar por temporadas.

Outro ponto de vista bastante importante é de que o estudo beneficia as descobertas de novos talentos, através dos testes trazendo resultados e mostrando assim destaques nas modalidades que podem mais á frente ser novas descorbertas em meio à modalidade. 
No decorrer do estudo foram encontradas algumas limitações, dentre elas, a principal limitação foi a quantidade reduzida de atletas para serem avaliados tornando a amostra do estudo bastante reduzida.

\section{CONCLUSÃO}

O presente estudo através da elaboração e validação de testes de agilidade e velocidade com atletas de voleibol sentado, veio por sua vez somar através de beneficio e fácil aplicabilidade dos testes com atletas da modalidade citada. Trazendo assim, uma maneira de avaliar e preparar as equipes da modalidade e dentre outras modalidades adaptadas, facilitando o trabalho dos profissionais e treinadores ligados a essa área, levando-os além da avaliação também a obter novas descobertas de futuros atletas.

\section{REFERÊNCIAS}

ADAMS, R; DANIEL, A; Mc CUBBIN, J. Jogos, Esportes e Exercícios para o Deficiente Físico. Trad. de Ângela Marx. 3.ed. São Paulo: Manole, 1985

ALEXANDRE, N. M. C.; COLUCI, M. Z. O. Validade de conteúdo nos processos de construção e adaptação de instrumentos de medidas.[Content validity in the development and adaptation processes of measurement instruments]. Ciência \& Saúde Coletiva, v. 16, n. 7, p. 3061-3068, 2011

BRASILE, F. Performance evaluation of wheelchair athletes: more than a disability classification level issue. Adapted Physical Activity Quarterly, Champaign, v.7, p.289-97, 1990.

BRAZUNA, M. R.; CASTRO, E. M. A trajetória do atleta portador de deficiência física no esporte adaptado de rendimento: uma revisão da literatura. Revista Motriz, Rio Claro, v. 7, n.2, p. 115-123,jul-dez, 2001.

CHAN, M; ZOELLICK, R. B.; Relatório Mundial Sobre a Deficiência. World Health Organization, The World Bank ; tradução Lexicus Serviços Lingüísticos. - São Paulo : SEDPcD, 2012. 334 p. ISBN 978-85-64047-02-0, 2011.

COMITÊ PARAOLÍMPICO BRASILEIRO - CPB. http://www.cpb.org.br/. Acesso em: 27/12/2017

COLLET, C.; NASCIMENTO, J. V.; RAMOS, V.; STEFANELLO, J. M. F. Construção e validação do instrumento de avaliação do desempenho técnico-tático no voleibol. Revista Brasileira de Cineantropometria e Desempenho Humano, Florianópolis, v. 13, n. 1, p. 43-51, 2011.

MELLO, M. T.; WINCKLER, C. (Org.). Esporte Paralímpico. 1ed. São Paulo: Editora Atheneu, 2012.

MOLIK, B., KOSMOL, A., \& SKUCAS, K.Sport - specific and general sporting physical fitness of sitting volleyball athletes. Physiotherapy, v. 16, n. 4, 68-75, 2008.

PEDRINELLI, V. J. Educação Física Adaptada: conceituação e terminologia. Educação Física e Desporto para Pessoas Portadoras de Deficiência. Brasília: MEC; SEDES; SESI-DN, 1994. p. 7-10.

SINGHAL, K. L. S. et al. Effects of Two Starting Floor Hand Positions on Movement Patterns of Elite Sitting Volleyball Players. Palestra, v. 27, n. 2, 2013.

SANTOS, A.C.; CHAGAS, E.F. Avaliação da aptidão neuromuscular e cardiorrespiratória dos integrantes da equipe de basquete em cadeira de rodas da FCT-UNESP. Congresso Brasileiro de Atividade Motora Adaptada, 4., Curitiba, 2001. Anais. Curitiba: SOBAMA/UFPR, 2001

WINNICK, J. P. Educação física e esportes adaptados. 3. ed. Barueri: Manole, 2004. 